\title{
Análise simultânea dos conceitos de ansiedade e medo: contribuições para os diagnósticos de enfermagem
}

Simultaneous analysis of the concepts of anxiety and fear: contributions to nursing diagnoses Análisis simultáneo de los conceptos de ansiedad y temor: contribuciones a los diagnósticos de enfermería

Claudia Angélica Mainenti Ferreira Mercês ${ }^{1,2}$ (1)

Jaqueline da Silva Soares Souto ${ }^{2}$ (1)

Priscilla Alfradique de Souza ${ }^{3}$ (1)

Marléa Crescêncio Chagas ${ }^{4}$ (D)

Carleara Weiss ${ }^{5}$ (1)

Andrea Barroso Benevides ${ }^{6}$ (I)

Marcos Antônio Gomes Brandão7 (1)

1. Instituto Nacional de Câncer José Alencar

Gomes da Silva. Rio de Janeiro, RJ, Brasil.

2. Universidade Federal do Rio de Janeiro.

Escola de Enfermagem Anna Nery, Programa de Pós-Graduação em Enfermagem. Rio de Janeiro, RJ, Brasil.

3. Universidade Federal do Estado do Rio de Janeiro, Escola de Enfermagem Alfredo Pinto, Departamento de Enfermagem MédicoCirúrgica. Rio de Janeiro, RJ, Brasil.

4. Universidade Federal do Rio de Janeiro, Escola de Enfermagem Anna Nery, Departamento de Metodologia de Enfermagem. Rio de Janeiro, RJ, Brasil.

5. University at Buffalo, Jacobs School of Medicine and Biomedical Sciences, Department of Pharmacology and Toxicology. New York, United States of America.

6. Universidade Federal do Rio de Janeiro, Escola de Enfermagem Anna Nery. Rio de Janeiro, RJ, Brasil.

7. Universidade Federal do Rio de Janeiro, Escola de Enfermagem Anna Nery, Departamento de Enfermagem Fundamental. Rio de Janeiro, RJ, Brasil.

Autor correspondente:

Claudia Angélica Mainenti Ferreira Mercês. E-mail: c.mainenti@globo.com.

Recebido em 10/06/2020.

Aprovado em 25/08/2020.

DOI:https://doi.org/10.1590/2177-9465-EAN-2020-0189

\section{Resumo}

Objetivos: Realizar a análise simultânea dos conceitos de ansiedade e medo. Método: Análise simultânea de conceitos com base nas nove etapas propostas por Haase, Leidy, Coward, Britt e Penn. Resultados: 43 artigos foram incluídos a partir de uma busca em cinco bases de dados. 51 atributos, 26 antecedentes e 10 consequentes foram identificados a partir da análise destes conceitos. Esta análise revelou que a ansiedade e o medo são conceitos subjetivos, multidimensionais e com altos graus de abstração. Conclusão: A análise simultânea da ansiedade e do medo possibilitou o refinamento destes diagnósticos e a proposição de modificações em seus componentes. Implicações para a prática: A análise destes conceitos possibilitará a identificação precisa destes fenômenos na prática clínica, proporcionando maior acurácia diagnóstica.

Palavras-chave: Ansiedade; Medo; Diagnóstico de enfermagem; Formação de conceito. Enfermagem.

\begin{abstract}
Objectives: To make a simultaneous analysis on the concepts of anxiety and fear. Method: Simultaneous concept analysis with nine steps proposed by Haase, Leidy, Coward, Britt, and Penn. Results: A total of 43 articles were included from a search in five literature databases. 51 attributes, 26 antecedents, and 10 consequents were identified from the analysis of these concepts. This analysis revealed that anxiety and fear are subjective, multidimensional concepts with high abstraction degrees. Conclusion: The simultaneous analysis of anxiety and fear is useful to refine these diagnoses and propose changes in their components. Implications for practice: The analysis of these concepts enables the precise identification of these effects in the clinical practice, applying greater diagnostic accuracy.
\end{abstract}

Keywords: Anxiety; Fear; Nursing diagnosis; Concept formation; Nursing.

\section{RESUMEN}

Objetivos: Realizar un análisis simultáneo de los conceptos de ansiedad y miedo. Método: Análisis simultáneo de conceptos basado en los nueve pasos propuestos por Haase, Leidy, Coward, Britt y Penn. Resultados: Se incluyeron 43 artículos de una búsqueda en cinco bases de datos. Del análisis de estos conceptos se identificaron 51 atributos, 26 antecedentes y 10 consecuencias. Este análisis reveló que la ansiedad y el miedo son conceptos subjetivos y multidimensionales con altos grados de abstracción. Conclusión: El análisis simultáneo de la ansiedad y del miedo permitió refinar estos diagnósticos y proponer cambios en sus componentes. Implicaciones para la práctica: el análisis de estos conceptos permitirá la identificación precisa de estos fenómenos en la práctica clínica, proporcionando una mayor precisión diagnóstica.

Palabras clave: Ansiedad; Miedo; Diagnóstico de Enfermería; Formación de Concepto. Enfermería. 


\section{INTRODUÇÃO}

No contexto da saúde, o ato anestésico, ${ }^{1}$ o tratamento cirúrgico, a hospitalização com o afastamento de familiares e modificações de atividades de vida, ${ }^{2}$ recebimento de diagnósticos e tratamentos diversos, principalmente de doenças associadas à terminalidade ${ }^{3}$ são fenômenos frequentemente considerados preditores positivos para a ocorrência da ansiedade e do medo. Estudos epidemiológicos, ${ }^{4}$ revisões sistemáticas e metaanálises ${ }^{5,6}$ identificaram $19 \%$ dos pacientes com níveis clínicos de ansiedade, 23\% com níveis subclínicos de ansiedade, e de 10 a $12 \%$ dos pacientes com câncer atendiam aos critérios para os tipos de transtorno de ansiedade. Nos contextos de atenção oncológica, Ansiedade e Medo são fenômenos complexos e comuns, identificados como diagnósticos de enfermagem ${ }^{7,8}$ ou denotando critérios relevantes ou características definidoras para o diagnóstico de enfermagem conforto prejudicado da NANDA-I. ${ }^{9}$

No campo da neurociência, distinções têm sido propostas entre as respostas comportamentais e fisiológicas defensivas ligadas a uma ameaça e os sentimentos dela decorrentes. No modelo de dois sistemas, o envolvimento de áreas corticais gera sentimentos conscientes diante da ameaça, ao passo que as regiões subcorticais operariam principalmente de forma inconsciente em comportamentos e respostas fisiológicas. A distinção entre o medo e ansiedade como sentimento consciente (sistema um) ou resposta fisiológica e comportamental inconsciente (sistema dois) é tão relevante quanto distinguir o medo da ansiedade. ${ }^{10}$

Como construtos diagnósticos de enfermagem, Medo (00148) e Ansiedade (00146) são distintos e dispostos pela NANDA-I. no domínio de "Enfrentamento e tolerância ao estresse". O diagnóstico de ansiedade, criado em 1973 e revisado em 1982 , 1998 e 2017, é definido como um "sentimento vago e incômodo de desconforto ou temor, acompanhado de resposta autonômica (fonte frequentemente não específica ou desconhecida pelo indivíduo), sentimento de apreensão causado pela antecipação do perigo, e é considerado um sinal de alerta que chama a atenção para um perigo iminente, o que permite ao indivíduo tomar medidas para lidar com a ameaça". O diagnóstico de medo, criado em 1980 e revisado em 1996, 2000 e 2017, é definido como uma "resposta à ameaça percebida que é conscientemente reconhecida como um perigo". ${ }^{11}$

Apesar das diferenças nas definições diagnósticas, Ansiedade e Medo são duas respostas humanas com indicadores clínicos compartilhados que podem estar presentes em ambos os diagnósticos, atuando como fator confundidor para a decisão clínica. Uma possível sobreposição na descrição conceitual pode existir e justificar a diferenciação dos conceitos.

Diferenciar um construto diagnóstico de outro pode ser uma tarefa extremamente difícil, sendo imprescindível considerar cuidadosamente as suas definições e as manifestações do paciente. ${ }^{12}$ Nessa perspectiva, Lunney destacou que enfermeiros podem desconhecer as diferenças entre o medo e a ansiedade, e desse modo, os utilizariam como se tivessem o mesmo significado. ${ }^{13}$
Contudo, terminologicamente o medo pode ser associado a uma ameaça ou perigo iminente e imediato, que desencadeia uma resposta imediata de luta ou fuga e geralmente desaparece quando a sugestão indutora do medo é removida; enquanto a ansiedade é referida a uma fonte de perigo ou ameaça incerta, afastada no tempo e espaço, e tendendo a durar mais tempo do que as respostas do medo. ${ }^{10,14}$

Independentemente do medo e ansiedade representarem um sentimento, emoção, ou resposta fisiológica e comportamental, para o construto diagnóstico de enfermagem, ambos fenômenos passam a ser representados como respostas humanas passíveis de intervenções por parte do enfermeiro. Frente a isso, estudos anteriores refinaram características definidoras dos diagnósticos de enfermagem de ansiedade e medo com validação com experts, ${ }^{15-17}$ analisaram conceitualmente a ansiedade e medo ${ }^{18} \mathrm{e}$ o medo de forma isolada. ${ }^{19}$ Pesquisadores utilizam a análise de conceito como uma metodologia para clarificar conceitos, diagnósticos e suas definições teóricas. ${ }^{20,21}$ No entanto, revisões anteriores falharam em distinguir as duas construções, exigindo uma investigação mais aprofundada. Ainda mais, considerando-se que conceitos e conhecimento são dinâmicos, é recomendável a constante atualização conceitual dos diagnósticos de enfermagem.

Neste intento, o objetivo do estudo é: realizar a análise simultânea dos conceitos de ansiedade e medo.

\section{MÉTODO}

Estudo de análise conceitual baseada no modelo de análise simultânea de conceitos. ${ }^{22} \mathrm{O}$ modelo possui nove etapas: desenvolvimento de um grupo consenso, seleção dos conceitos que serão analisados, refinamento da abordagem de clarificação dos conceitos, clarificação dos conceitos individuais, desenvolvimento de matrizes de validação, revisão dos conceitos individuais, reexame das matrizes de validação, desenvolvimento de um modelo processual e submissão dos resultados da análise simultânea dos conceitos aos especialistas para crítica.

Análises de conceitos são estudos de validação diagnóstica de enfermagem que antecedem a validação por experts e a validação clínica. ${ }^{23} \mathrm{Na}$ atualidade, os diagnósticos de Medo (00148) e Ansiedade (000146) não estão ancorados em nenhum nível de evidência. Por este motivo, a NANDA International, Inc. em sua última edição encoraja o desenvolvimento de estudos, principalmente com diagnósticos mais antigos, com objetivo de fornecer níveis de evidências aos mesmos, para que possam ser mantidos na terminologia. ${ }^{11}$

Estudos de análise de conceito têm em sua maioria utilizado a abordagem mais clássica proposta por Lorraine O. Walker e Kay C. Avant. Entretanto, esta abordagem não serve ao propósito de diferenciar conceitos similares ou inter-relacionados. Para tanto, Haase, Leidy, Coward, Britt e Penn propuseram a estratégia de análise simultânea de conceitos, na qual ocorre a análise individual de dois ou mais conceitos, sendo acompanhadas de um exame crítico das inter-relações dos antecedentes, consequentes e atributos críticos. 


\section{Etapas 1 e 2 - Desenvolvimento do grupo consenso e seleção de conceitos a serem analisados}

O grupo consenso foi formado por três pesquisadores. Um dos pesquisadores atuou como analista e moderador do grupo consenso. Todos os participantes tinham conhecimento prévio sobre métodos de análise de conceito.

A seleção dos conceitos de ansiedade e medo foi baseada na relevância clínica que estes fenômenos representam nos diversos contextos de saúde e pela ausência de estudos analíticos recentes desses dois conceitos de forma simultânea.

\section{Etapa 3 - Refinamento da abordagem de clarificação dos conceitos}

Dando cumprimento à terceira etapa, foi construído por uma das autoras um instrumento para extração dos antecedentes, atributos, consequentes e definições para cada um dos conceitos. A mesma autora também realizou uma revisão integrativa, ${ }^{24} \mathrm{sem}$ estabelecimento de um recorte temporal. A busca foi desenvolvida no mês de abril de 2017, por meio do acesso às seguintes bases de dados: Literatura Latino-Americana e do Caribe em Ciências da Saúde (LILACS), Medical Literature Analysis and Retrieval Sistem on Line (MEDLINE), Cummulative Index to Nursing and Allies Health Literature (CINAHL), Excerpta medica Database (EMBASE) e Behavioral Sciences and Mental Health (PSYCINFO).

Os termos de busca utilizados nas bases de dados foram "sinais e sintomas", "ansiedade", "medo", "antecedentes" e "consequências", combinados com os operadores booleanos
AND e OR. Os critérios de inclusão foram estudos qualitativos, quantitativos ou mistos, publicados nos seguintes idiomas: português, inglês ou espanhol. E os critérios de exclusão foram: artigos duplicados, não adesão ao tema proposto, relatos de experiências, revisões de literatura, editoriais e cartas ao editor.

O processo de busca foi realizado da seguinte maneira: aplicação das estratégias nas bases de dados selecionadas; remoção das duplicatas; aplicação dos critérios de inclusão e exclusão na leitura de títulos e resumos; seleção e leitura dos textos completos; e leitura e análise dos textos selecionados por três pesquisadores. ${ }^{24}$ Os textos selecionados foram arquivados no gerenciador bibliográfico EndNote. Ao final desse processo foram selecionados artigos que compuseram a amostra final desta revisão (vide Figura 1).

Para a análise do conceito a revisão integrativa representa a técnica, e não o método, portanto, o presente estudo prossegue no detalhamento dos procedimentos analíticos.

\section{Etapa 4 - Clarificação dos conceitos individuais}

Na quarta etapa, o moderador do grupo consenso analisou os textos selecionados referentes aos conceitos de ansiedade e medo e extraiu todos os elementos de decomposição conceitual e os alocou no referido instrumento. Dois analistas realizaram o mesmo procedimento, porém cada um ficou responsável por um dos conceitos.

Após as análises individuais, todas foram reunidas e cada atributo, antecedente e consequente só foi considerado apropriado para inclusão quando obteve a concordância dos três

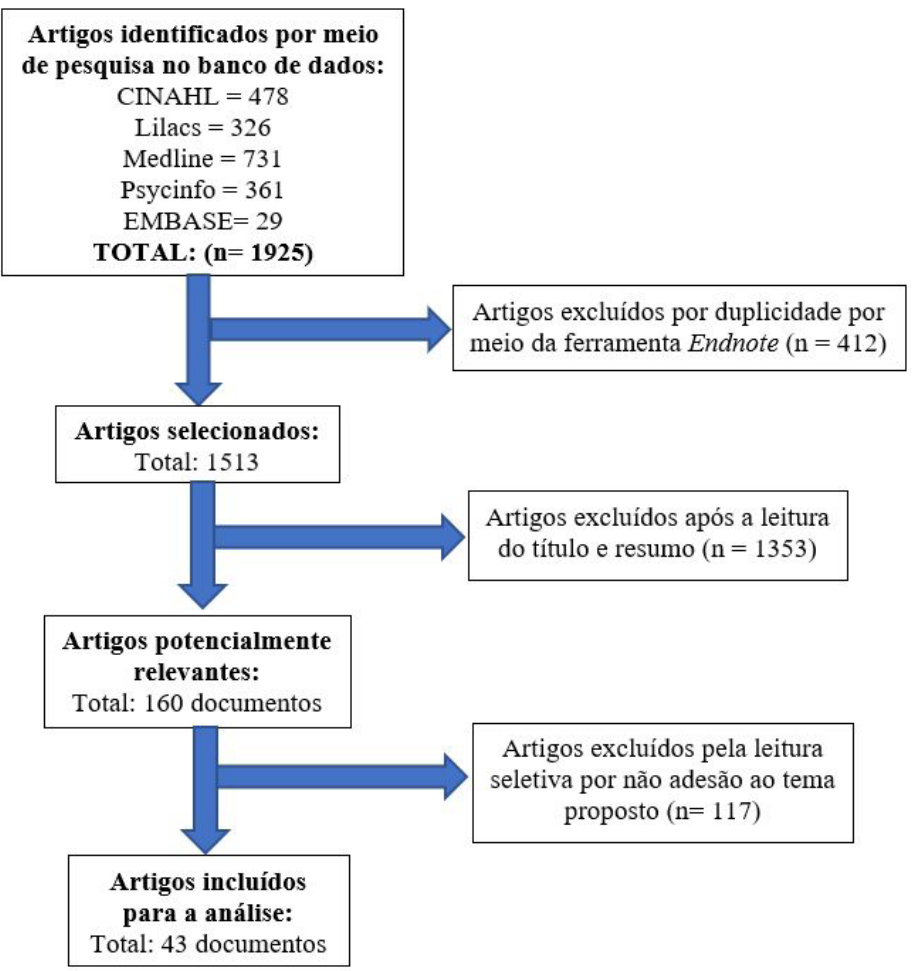

Figura 1. Fluxograma de estudos identificados e selecionados para inclusão na revisão integrativa. Fonte: Elaborado pelos autores. 
integrantes do grupo consenso. Tal procedimento tem o escopo de aumentar a confiabilidade do estudo, reduzindo o possível viés de interpretação individual.

\section{Etapa 5 - Desenvolvimento de matrizes de validação}

$\mathrm{Na}$ quinta etapa, foram construídas matrizes de validação para os antecedentes, atributos e consequentes de cada um dos conceitos. Sendo possível visualizar as diferenças e inter-relações de cada conceito, oferecendo uma visão sobre o potencial de confusão e sobreposição teórica subjacente a cada conceito.

\section{Etapas 6 e 7 - Revisão dos conceitos individuais e reexame das matrizes de validação}

Dando prosseguimento, nas etapas 6 e 7 foi realizado um reexame de todos os conceitos e das matrizes de validação, no intento de evitar a inclusão de algum elemento que não fosse necessário.

\section{Etapa 8 - Desenvolvimento de um Modelo Processual}

$\mathrm{Na}$ oitava etapa, um modelo processual do medo e da ansiedade foi construído, para possibilitar a visualização em uma única imagem de todos os elementos constitutivos para formação de conceito.

\section{Etapa 9 - Submissão dos resultados da análise simultânea de conceitos aos especialistas para crítica}

A nona etapa foi a submissão dos resultados da análise simultânea dos conceitos de ansiedade e medo a três enfermeiros especialistas em oncologia, com base na premissa de que o medo e a ansiedade são frequentemente verificados em suas práticas clínicas. ${ }^{3,25}$ A seleção dos especialistas seguiu os seguintes critérios: fazer parte do quadro permanente de funcionários de um hospital público federal localizado na cidade do Rio de Janeiro especializado em oncologia; ter no mínimo cinco anos de experiência clínica na área; e ter o título de especialista. O objetivo desta etapa foi a avaliação externa do modelo. Por fim, por consenso, o modelo foi aprovado. Esta etapa não deve ser confundida com a validação por especialistas, pois representa uma amostra reduzida, descrita no método. A experiência dos enfermeiros de oncologia foi usada como uma avaliação de qualidade nesta etapa.

\section{RESULTADOS}

\section{Antecedentes}

A análise dos antecedentes possibilitou identificar 0 conhecimento produzido acerca dos fatores que antecedem os fenômenos da ansiedade e do medo e verificar se estes estão presentes nos diagnósticos de enfermagem de "Ansiedade" e "Medo" inseridos na taxonomia NANDA-I, comparando-os aos fatores relacionados, populações em risco e condições associadas.
O consenso dos analistas permitiu a incorporação de 26 antecedentes para os conceitos de ansiedade e medo. Destes, 7 são exclusivos ao conceito de ansiedade, 12 são compartilhados pelos dois conceitos e 7 são exclusivos ao conceito de medo.

Dos 19 antecedentes identificados para o conceito de ansiedade, 13 foram identificados a partir do consenso e não fazem parte dos constituintes atuais da NANDA- I. Já para o conceito de medo, foram identificados também 19 antecedentes, mas destes 15 não estão presentes na NANDA-I.

\section{Atributos}

O consenso dos especialistas permitiu identificar a incorporação de 51 atributos para os conceitos de ansiedade e medo. Destes, 11 são exclusivos à ansiedade, 29 são compartilhados pelos dois conceitos e 11 são exclusivos ao medo. Estes atributos foram comparados às características definidoras presentes na NANDA-I.

Dos 40 atributos presentes para o conceito de ansiedade, 13 não estão presentes na NANDA-I. E para os 40 atributos do medo, 21 não estão presentes na NANDA- I.

\section{Consequentes}

Para Walker e Avant, em uma análise de conceito, os consequentes são aqueles eventos ou incidentes que ocorrem como resultado da ocorrência do conceito, ou seja, são os resultados do conceito. ${ }^{26}$

As consequências foram classificadas como negativas nesta análise simultânea, com base no entendimento de que as consequências carregam a dimensão de resultados ou objetivos a serem alcançados com ou sem a resolução dos problemas. Consequências negativas implicam que elas podem impor danos psicológicos, fisiológicos e sociais aos sujeitos. Espera-se que sejam modificados a partir da seleção e implementação do cuidado de enfermagem assertivo. Os resultados das etapas 5 a 9 são descritos na Tabela 1.

\section{DISCUSSÃO}

Os resultados provenientes da análise simultânea dos conceitos de ansiedade e medo revelaram que estes são conceitos subjetivos, multidimensionais e com alto grau de abstração. Foram caracterizados como reações, emoções, estados, sentimentos, experiências e englobam aspectos emocionais, fisiológicos, psicológicos, sociais, cognitivos e culturais. São bastante frequentes nos contextos da prática clínica de enfermagem, sendo difíceis de serem diferenciados e, portanto, difíceis de serem operacionalizados.

O modelo processual refletiu características distintas de cada um dos dois conceitos, já que em uma única imagem é possível visualizar as fontes potenciais de confusão dos dois conceitos, como por exemplo, o grande compartilhamento de atributos entre os conceitos, assim como é possível obter uma imagem do que é exclusivo à cada conceito, conferindo-lhe uma identidade. Ainda sobre este modelo processual, é possível afirmar que há sinonímias, inter-relações e diferenças entre os conceitos. 
Isto contribui também para o desenvolvimento de definições que são únicas para cada conceito e ao mesmo tempo são destacadas as inter-relações e os elementos distintos dos mesmos que estão associados aos conceitos, possibilitando a identificação do que é exclusivo ao conceito, ou seja, sua identidade e o que é compartilhado por dois ou mais conceitos. O objetivo principal desta estratégia é obter uma visão das fontes potenciais de confusão teórica, empírica e prática entre os conceitos, assim como oferecer melhor compreensão do processo que constitui a base de cada conceito. ${ }^{22}$

A última etapa da análise simultânea, que é a submissão dos resultados da análise simultânea aos pares para crítica, não deve ser entendida como um estudo de validação de conteúdo por especialistas. Os enfermeiros especialistas em oncologia avaliaram os resultados, já que a ansiedade e o medo são frequentemente vivenciados por pacientes com doenças

Tabela 1. Matriz de validação e modelo processual para os antecedentes, atributos e consequentes dos conceitos de ansiedade e medo.

\begin{tabular}{|c|c|c|c|}
\hline & ANSIEDADE & ANSIEDADE E MEDO & MEDO \\
\hline ANTECEDENTES & $\begin{array}{l}\text { Abuso de } \\
\text { substâncias; }{ }^{27-30} \text { baixa } \\
\text { autoestima;,19,31-33 } \\
\text { hospitalizações } \\
\text { anteriores; }{ }^{34,35} \text { solidão; }{ }^{30} \\
\text { hereditariedade; } \\
\text { história familiar de } \\
\text { ansiedade; ; } 2731,37 \\
\text { história pregressa de } \\
\text { ansiedade. }{ }^{36,38-42}\end{array}$ & $\begin{array}{l}\text { Ambiente desconhecido; }{ }^{1,18,34,35} \text { Ameaça } \\
\text { à condição atual; }{ }^{18,43} \text { Conhecimento } \\
\text { deficiente; }{ }^{19,41} \text { Estressores; }{ }^{1,18,28,38,39,42,44,45} \\
\text { Ameaça de morte; }{ }^{46} \text { Incerteza; }{ }^{41,42} \\
\text { Separação do sistema de apoio; }{ }^{19,34,38,47} \\
\text { Idade jovem; }{ }^{27,37,40,41,48-50} \text { Sexo } \\
\text { feminino; }{ }^{27,32,37,46,48,50-52} \text { Diagnóstico } \\
\text { de uma doença; }{ }^{: 28,41,53} \text { Procedimentos } \\
{\text { clínicos e cirúrgicos; }{ }^{1,28,35} \text { Sintomas físicos }}^{\text {de uma doença. }{ }^{46,50}}\end{array}$ & $\begin{array}{l}\text { Barreira linguística; }{ }^{19,47} \text { Condições } \\
\text { inatas; }{ }^{: 18,19} \text { Percepção do risco de } \\
\text { recidiva da doença; }{ }^{42,49,51} \text { Situações } \\
\text { de vulnerabilidade; }{ }^{19,47,54} \text { Baixa } \\
\text { escolaridade; }{ }^{40,45} \text { Baixo nível sócio } \\
\text { econômico; }{ }^{45} \text { Déficit sensorial. }{ }^{19}\end{array}$ \\
\hline ATRIBUTOS & $\begin{array}{l}\text { Alteração do } \\
\text { humor; }{ }^{31,43,44,46} \\
\text { Impotência; }{ }^{35,52,55} \\
\text { Sensação de solidão; }{ }^{34,35} \\
\text { Tristeza; }{ }^{34,35,55} \text { Foco } \\
\text { em si próprio; }{ }^{16,18,56} \\
\text { Alteração do apetite; }{ }^{35} \\
\text { Cefaleia;; }{ }^{27,31,43} \\
\text { Deglutição } \\
\text { prejudicada; }{ }^{46} \\
\text { Fadiga; }{ }^{19,27,30,41,43,57,58} \\
\text { Náuseas e } \\
\text { vômitos; }{ }^{19,31,39,43} \\
\text { Tonteira. } .^{31,55-57}\end{array}$ & 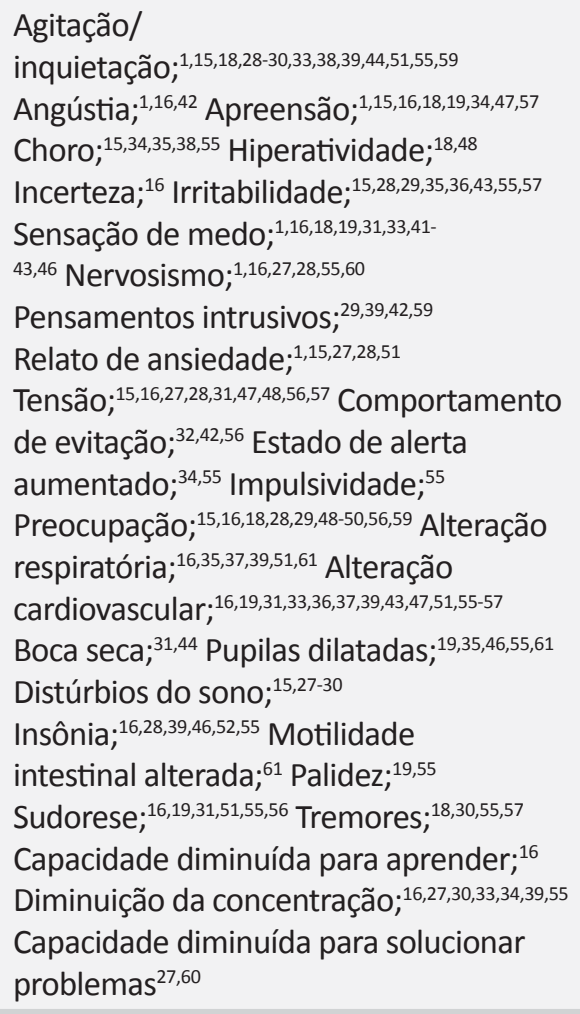 & $\begin{array}{l}\text { Sensação de pânico; }{ }^{15,16,19} \\
\text { Sofrimento; }{ }^{34,59} \text { Sensação } \\
\text { de terror; }{ }^{19,57} \text { Aversão; }{ }^{22,63} \\
\text { Comportamento de fuga; }{ }^{18,19,55,61} \\
\text { Comportamento de luta; }{ }^{18,19,55,61} \\
\text { Congelamento/paralisia; }{ }^{55,61} \text { Foco } \\
\text { na fonte de ameaça; }{ }^{18} \text { Aumento da } \\
\text { glicemia capilar; }{ }^{61} \text { Diminuição da } \\
\text { produtividade; }{ }^{16} \text { Identifica o objeto } \\
\text { do medo. }{ }^{61}\end{array}$ \\
\hline CONSEQUENTES & $\begin{array}{l}\text { Baixa produtividade; }{ }^{57} \\
\text { Dependência de } \\
\text { substâncias; }{ }^{44,47} \\
\text { Sistema imunológico } \\
\text { prejudicado; }{ }^{44} \\
\text { Surgimento de } \\
\text { comorbidades. }{ }^{18,51,64}\end{array}$ & $\begin{array}{l}\text { Aumento de gastos com a saúde; }{ }^{42,43,53} \\
\text { Baixa qualidade de vida; } 77,42,49,51,52,64 \\
\text { Declínio da saúde física; }{ }^{36,39,44,51} \\
\text { Distúrbios alimentares; }{ }^{44} \text { Transtornos } \\
\text { psicológicos. }{ }^{32,39,44,49}\end{array}$ & $\begin{array}{l}\text { Memória do medo de longa } \\
\text { duração. }{ }^{18}\end{array}$ \\
\hline
\end{tabular}


associadas à terminalidade, como o câncer, ${ }^{3}$ o que não exclui que este modelo também seja avaliado por enfermeiros de outras especialidades.

Cada especialista recebeu uma cópia do modelo processual, uma cópia de todas as definições conceituais dos antecedentes, atributos e consequentes e um instrumento com uma escala likert, com oito indicadores visando verificar o grau de concordância dos especialistas quanto ao modelo processual, no que se refere à: consistência em representar os conceitos, capacidade de demonstrar as similaridades e diferenças entre os conceitos, estabelecimento de relações entre os conceitos, adequação e suficiência dos termos, layout e adequação para uso na prática clínica da enfermagem oncológica.

Todos especialistas concordaram com a adequação do modelo processual na prática clínica, além de destacarem que o mesmo foi capaz de estabelecer as diferenças e similitudes entre os conceitos.

A análise simultânea dos conceitos possibilitou a proposição de uma nova composição diagnóstica para os diagnósticos de "Ansiedade" e "Medo". Sendo possível então propor uma definição para cada diagnóstico de enfermagem, bem como a inclusão de alguns antecedentes e atributos que estão fortemente associados aos conceitos e que auxiliam na identificação precisa do fenômeno, porém não estão presentes na classificação da Nanda-I. Foi possível também sugerir a substituição de alguns termos existentes. Os antecedentes são representados pelos fatores relacionados, e também pelas populações em risco e condições associadas. Assim como os atributos são interpretados pelas características definidoras.

Por meio da análise foi possível propor uma nova definição para o diagnóstico de enfermagem de "Ansiedade" (00146), ou seja, substituir "Sentimento vago e incômodo de desconforto ou temor, acompanhado por resposta autonômica (a fonte é frequentemente não específica ou desconhecida para o indivíduo); sentimento de apreensão causado pela antecipação do perigo. É um sinal de alerta que chama a atenção para um perigo iminente e permite ao indivíduo tomar medidas para lidar com a ameaça"11 por "Sentimento provocado por uma antecipação de uma ameaça futura que frequentemente é inespecífica ou desconhecida e apresenta componentes psicológicos, fisiológicos, comportamentais e cognitivos de caráter mais duradouro".

Quanto às substituições, não houve nenhuma substituição dos antecedentes. Entretanto, sugerimos a substituição de alguns atributos, conforme descrito no Quadro 1. Esse procedimento

Quadro 1. Proposta de reformulação dos diagnósticos de enfermagem de "Ansiedade" e "Medo".

\begin{tabular}{|c|c|c|c|c|}
\hline Título & \multicolumn{4}{|c|}{ Ansiedade } \\
\hline Domínio & \multicolumn{4}{|c|}{ Domínio 9 - Enfrentamento/Tolerância ao estresse } \\
\hline Definição & \multicolumn{4}{|c|}{$\begin{array}{l}\text { Sentimento provocado por antecipação de uma ameaça futura que frequentemente é inespecífica ou } \\
\text { desconhecida e apresenta componentes psicológicos, fisiológicos, comportamentais e cognitivos de } \\
\text { caráter mais duradouro. }\end{array}$} \\
\hline $\begin{array}{c}\text { Características } \\
\text { Definidoras }\end{array}$ & $\begin{array}{l}\text { Afetivas: Agitação/ } \\
\text { inquietação (b); } \\
\text { Agonia; Alteração do } \\
\text { humor (a); Angústia } \\
\text { (a); Apreensão; } \\
\text { Arrependimento; } \\
\text { Choro (a); Desamparo; } \\
\text { Entusiasmo excessivo; } \\
\text { Hiperatividade (a); } \\
\text { Impotência (a); } \\
\text { Incerteza; Irritabilidade; } \\
\text { Nervosismo; } \\
\text { Pensamentos intrusivos } \\
\text { (b); Relato de } \\
\text { ansiedade (a); Sensação } \\
\text { de inadequação; } \\
\text { Sensação de medo (b); } \\
\text { Sensação de solidão } \\
\text { (a); Sofrimento; } \\
\text { Suspeição aumentada; } \\
\text { Tensão (b); Tristeza (a). }\end{array}$ & $\begin{array}{l}\text { Cognitivas: Bloqueio } \\
\text { de pensamentos; } \\
\text { Campo de percepção } \\
\text { diminuído; Capacidade } \\
\text { diminuída para } \\
\text { aprender; Capacidade } \\
\text { diminuída para } \\
\text { solucionar problemas; } \\
\text { Confusão; Consciência } \\
\text { dos sintomas } \\
\text { fisiológicos; Diminuição } \\
\text { da concentração } \\
\text { (b); Esquecimento; } \\
\text { Tendência a culpar os } \\
\text { outros. }\end{array}$ & $\begin{array}{l}\text { Comportamentais: Age } \\
\text { como se examinasse o } \\
\text { ambiente; Atos bruscos; } \\
\text { Comportamento de } \\
\text { evitação (b); Estado de } \\
\text { alerta (b); Foco em si } \\
\text { próprio; Impulsividade } \\
\text { (a); Movimento sem } \\
\text { finalidade; Movimentos } \\
\text { bruscos; Preocupação } \\
\text { (b); Produtividade } \\
\text { diminuída; Reflexos } \\
\text { rápidos. }\end{array}$ & $\begin{array}{l}\text { Fisiológicas: Alteração } \\
\text { cardiovascular (b); } \\
\text { Alteração da pressão } \\
\text { arterial (b); Alteração } \\
\text { do apetite (b); Alteração } \\
\text { respiratória (b); Alteração } \\
\text { urinária (b); Boca seca; } \\
\text { Cefaleia (a); Deglutição } \\
\text { prejudicada (a); Desmaio; } \\
\text { Distúrbios do sono (b); } \\
\text { Dor abdominal; Fadiga; } \\
\text { Formigamento das } \\
\text { extremidades; Fraqueza; } \\
\text { Insônia; Motilidade } \\
\text { intestinal alterada (b); } \\
\text { Náuseas e vômitos } \\
\text { (b); Palidez (a); Pupilas } \\
\text { dilatadas; Rubor facial; } \\
\text { Sudorese (b); Tonteira (a) } \\
\text { Tremores; Vasoconstrição } \\
\text { superficial. }\end{array}$ \\
\hline
\end{tabular}

(a) Inclusão de um termo. (b) Substituição de um termo. 
Quadro 1. Continuação...

\begin{tabular}{|c|c|c|c|c|}
\hline Título & \multicolumn{4}{|c|}{ Ansiedade } \\
\hline $\begin{array}{c}\text { Fatores } \\
\text { Relacionados }\end{array}$ & \multicolumn{4}{|c|}{$\begin{array}{l}\text { Abuso de substâncias; Ambiente desconhecido (a); Ameaça à condição atual; Ameaça de morte; Baixa } \\
\text { autoestima (a); Conhecimento deficiente (a); Conflito de valores; Conflito sobre as metas da vida; } \\
\text { Contágio interpessoal; Estressores; Hospitalizações anteriores (a); Incerteza (a); Necessidades não } \\
\text { atendidas; Separação do sistema de apoio (a); Solidão (a); Transmissão interpessoal. }\end{array}$} \\
\hline $\begin{array}{l}\text { Populações em } \\
\text { Risco }\end{array}$ & \multicolumn{4}{|c|}{$\begin{array}{l}\text { Crise maturacional; Crise situacional; Exposição a toxinas; Hereditariedade; História familiar de } \\
\text { ansiedade; História pregressa de ansiedade (a); Idade jovem (a); Mudança importante; Sexo feminino } \\
\text { (a); }\end{array}$} \\
\hline $\begin{array}{r}\text { Condição } \\
\text { Associada }\end{array}$ & \multicolumn{4}{|c|}{$\begin{array}{l}\text { Diagnóstico de uma doença (a); Procedimentos clínicos e cirúrgicos (a); Sintomas físicos de uma } \\
\text { doença (a). }\end{array}$} \\
\hline Título & \multicolumn{4}{|l|}{ Medo } \\
\hline Domínio & \multicolumn{4}{|c|}{ Domínio 9 - Enfrentamento/Tolerância ao estresse } \\
\hline Definição & \multicolumn{4}{|c|}{$\begin{array}{l}\text { Resposta imediata à uma ameaça que é conscientemente reconhecida como um perigo, estando } \\
\text { associada ao comportamento de fuga ou luta }\end{array}$} \\
\hline $\begin{array}{l}\text { Características } \\
\text { Definidoras }\end{array}$ & $\begin{array}{l}\text { Afetivas: Agitação/ } \\
\text { inquietação } \\
\text { (b); Angústia } \\
\text { (a); Apreensão; } \\
\text { Autossegurança } \\
\text { diminuída; Choro (a); } \\
\text { Hiperatividade (a); } \\
\text { Incerteza(a);Iritabilidade(a); } \\
\text { Nervosismo (a); } \\
\text { Pensamentos intrusivos } \\
\text { (a); Relato de } \\
\text { ansiedade (a); Sensação } \\
\text { de alarme; Sensação } \\
\text { de medo; Sensação de } \\
\text { pânico; Sensação de } \\
\text { receio; Sensação de } \\
\text { terror; Sofrimento (a); } \\
\text { Tensão (b). }\end{array}$ & $\begin{array}{l}\text { Cognitivas: Capacidade } \\
\text { de resolução de } \\
\text { problemas diminuída; } \\
\text { Capacidade } \\
\text { diminuída para } \\
\text { aprender (b); Diminuição } \\
\text { da concentração (a); } \\
\text { Diminuição da } \\
\text { produtividade (a); } \\
\text { Estímulos entendidos } \\
\text { como ameaça; } \\
\text { Identifica o objeto do } \\
\text { medo; Produtividade } \\
\text { diminuída. }\end{array}$ & $\begin{array}{l}\text { Comportamentais: } \\
\text { Aversão (a); } \\
\text { Comportamento } \\
\text { de evitação (a); } \\
\text { Comportamento } \\
\text { de fuga (b); } \\
\text { Comportamento de } \\
\text { luta (b); Congelamento/ } \\
\text { paralisia (a); Estado } \\
\text { de alerta aumentado; } \\
\text { Foco na fonte de } \\
\text { ameaça(b);Impulsividade(a); } \\
\text { Preocupação (a). }\end{array}$ & $\begin{array}{l}\text { Fisiológicas: Alteração } \\
\text { cardiovascular (a); } \\
\text { Alteração na resposta } \\
\text { fisiológica; Alteração do } \\
\text { apetite (b); Alteração } \\
\text { respiratória (b); } \\
\text { Aumento da glicemia } \\
\text { capilar (a); Aumento da } \\
\text { pressão arterial; Boca } \\
\text { seca; Distúrbios do sono } \\
\text { (a); Fadiga; Insônia (a); } \\
\text { Motilidade intestinal } \\
\text { alterada (b); Náuseas } \\
\text { e vômitos (b); Palidez; } \\
\text { Pupilas dilatadas; } \\
\text { Sudorese (b); Tremores } \\
\text { (a). }\end{array}$ \\
\hline $\begin{array}{c}\text { Fatores } \\
\text { Relacionados }\end{array}$ & \multicolumn{4}{|c|}{$\begin{array}{l}\text { Ambiente desconhecido (b); Ameaça à condição atual (a); Ameaça de morte (a); Barreira linguística; Condições } \\
\text { inatas (a); Conhecimento deficiente (a); Estressores (a); Incerteza (a); Percepção do risco de recidiva da doença } \\
\text { (a); Reação a um estímulo fóbico; Reação aprendida a uma ameaça; Separação do sistema de apoio; Situações } \\
\text { de vulnerabilidade (a). }\end{array}$} \\
\hline $\begin{array}{l}\text { Populações em } \\
\text { Risco }\end{array}$ & \multicolumn{4}{|c|}{ Baixa escolaridade (a); Baixo nível socioeconômico (a); Idade jovem (a); Sexo feminino (a). } \\
\hline $\begin{array}{r}\text { Condição } \\
\text { Associada }\end{array}$ & \multicolumn{4}{|c|}{$\begin{array}{l}\text { Déficit sensorial; Diagnóstico de uma doença (a); Procedimentos clínicos e cirúrgicos (a); Sintomas } \\
\text { físicos de uma doença (a). }\end{array}$} \\
\hline
\end{tabular}

(a) Inclusão de um termo. (b) Substituição de um termo.

objetiva reduzir a longa lista de indicadores clínicos pertencentes a um diagnóstico, que por vezes são muito similares e que acabam por dificultar o raciocínio diagnóstico. Já que a melhor análise é aquela que refina os atributos para o menor número possível e que permite diferenciar o conceito em questão dos outros circundantes. ${ }^{26}$

Para o conceito de medo, a análise realizada possibilitou a proposição de uma nova definição para o diagnóstico de enfermagem "Medo" (00148) que propõe substituir "resposta a uma ameaça percebida que é conscientemente reconhecida como um perigo"11 por "resposta imediata à uma ameaça que é conscientemente reconhecida como um perigo, estando associada ao comportamento de fuga ou luta".

Propõe-se apenas uma substituição dos antecedentes, e também a substituição de alguns atributos. O Quadro 1 sintetiza a proposta de conteúdo para os diagnósticos de enfermagem em questão, a partir da análise simultânea de conceito realizada. 


\section{CONCLUSÕES E IMPLICAÇÕES PARA A PRÁTICA}

A ansiedade e o medo são fenômenos frequentemente vivenciados por pacientes em diversos contextos de saúde, exigindo um claro entendimento destes conceitos pelo enfermeiro. A análise simultânea possibilitou a proposição de novas definições para estes diagnósticos presentes na taxonomia NANDA-I, a inclusão e a substituição de fatores relacionados, populações em risco, condições associadas e características definidoras, corroborando para uma melhor compreensão destes fenômenos.

O método utilizado evidenciou que a ansiedade e o medo são fenômenos multidimensionais, com alto nível de abstração. A importância deste estudo reside na clarificação destes conceitos, podendo auxiliar na precisão diagnóstica e consequentemente na implementação de cuidados de enfermagem mais efetivos.

Considerando que a linguagem diagnóstica desta taxonomia está em constante aperfeiçoamento e que diagnósticos sem níveis de evidências poderão ser retirados da taxonomia na próxima edição, a análise destes conceitos possibilita o fornecimento de níveis de evidências para os diagnósticos de ansiedade e medo conforme os critérios de níveis de evidências do comitê de Educação e pesquisa da NANDA-I.

Espera-se com este estudo contribuir para o avanço do conhecimento e o aperfeiçoamento da linguagem diagnóstica, ao clarificar estes fenômenos, destacar as suas similitudes e diferenças. Além disso, poderá auxiliar estudantes e enfermeiros na identificação precisa destes diagnósticos.

Uma das limitações deste estudo pode estar relacionada à seleção de textos apenas nos idiomas de português, espanhol e inglês, impossibilitando a inclusão de outras culturas. Outra limitação reside no fato de não ter sido proposto a retirada de alguns indicadores dos diagnósticos de enfermagem de Ansiedade e Medo presentes na NANDA-I e que não foram evidenciados durante a revisão integrativa.

Além disso, uma limitação à generalização da crítica ao modelo processual advém de somente enfermeiros especialistas em oncologia terem feito a apreciação do modelo. Estudos futuros podem explorar a opinião de enfermeiros de outras especialidades. Porém, esse limite não é um problema para a validade do estudo, considerando que em níveis de evidência, a revisão e análise feitas a partir da literatura superam a opinião de especialistas sobre o modelo processual.

\section{CONTRIBUIÇÕES DOS AUTORES}

Concepção do estudo: Claudia Angélica Mainenti Ferreira Mercês. Marcos Antônio Gomes Brandão

Levantamento das fontes de informações: Claudia Angélica Mainenti Ferreira Mercês. Jaqueline da Silva Soares Souto

Análise e Interpretação dos dados extraídos das fontes: Claudia Angélica Mainenti Ferreira Mercês. Jaqueline da Silva Soares Souto. Priscilla Alfradique de Souza. Marléa Crescêncio Chagas. Carleara Weiss. Andrea Barroso Benevides. Marcos Antônio Gomes Brandão
Redação e revisão crítica: Claudia Angélica Mainenti Ferreira Mercês. Jaqueline da Silva Soares Souto. Priscilla Alfradique de Souza. Marléa Crescêncio Chagas. Carleara Weiss. Andrea Barroso Benevides. Marcos Antônio Gomes Brandão

Aprovação da versão final do artigo: Claudia Angélica Mainenti Ferreira Mercês. Jaqueline da Silva Soares Souto. Priscilla Alfradique de Souza. Marléa Crescêncio Chagas. Carleara Weiss. Andrea Barroso Benevides. Marcos Antônio Gomes Brandão

Responsabilidade pelo conteúdo intelectual: Claudia Angélica Mainenti Ferreira Mercês. Jaqueline da Silva Soares Souto. Priscilla Alfradique de Souza. Marléa Crescêncio Chagas. Carleara Weiss. Andrea Barroso Benevides. Marcos Antônio Gomes Brandão

\section{EDITOR ASSOCIADO}

Stela Maris de Mello Padoin

\section{REFERÊNCIAS}

1. Suriano MLF, Lopes DCDF, Macedo GPDOS, Michel JLM, Barros ALBLD. Identification of the defining characteristics of fear and anxiety in patients scheduled for gynecological surgery. Acta Paul Enferm. 2009;22:928-34

2. Grisa GH, Monteiro JK. Aspectos emocionais do paciente cardíaco cirúrgico no período pré-operatório. Gerais [Internet]. 2015; [citado 2020 jun 10];8(1):111-30. Disponível em: https://pdfs.semanticscholar.org/ d8b5/3ab60f2882bc60f84dffbaa6aac580b87838.pdf

3. Bronner MB, Nguyen MH, Smets EM, Van de Ven AW, van Weert JC Anxiety during cancer diagnosis: Examining the influence of monitoring coping style and treatment plan. Psychooncology.2018;27(2):661-7.http:// dx.doi.org/10.1002/pon.4560. PMid:28976610.

4. Mehnert A, Brähler E, Faller H, Härter M, Keller M, Schulz H et al. Fourweek prevalence of mental disorders in patients with cancer across major tumor entities. J Clin Oncol.2014;32(31):3540-6. http://dx.doi.org/10.1200/ JCO.2014.56.0086. PMid:25287821.

5. Mitchell AJ, Chan M, Bhatti H, Halton M, Grassi L, Johansen C et al Prevalence of depression, anxiety, and adjustment disorder in oncological, haematological, and palliative-care settings: a meta-analysis of 94 interviewbased studies. Lancet Oncol.2011;12(2):160-74.http://dx.doi.org/10.1016/ S1470-2045(11)70002-X. PMid:21251875.

6. Vehling S, Koch U, Ladehoff N, Schön G, Wegscheider K, Heckl U et al Prevalence of affective and anxiety disorders in cancer: systematic literature review and meta-analysis. Psychother Psychosom Med Psychol. 2012;62(7):249-58. http://dx.doi.org/10.1055/s-0032-1309032. PMid:22585582

7. Sousa RMD, Santo FHDE, Santana RF, Lopes MVDO. Nursing diagnoses identified in onco-hematologic patients: a cross-mapping study. Esc Anna Nery. 2015;19(1):54-65. http://dx.doi.org/10.5935/1414-8145.20150008.

8. Castro MCF, Fuly PDSC, Garcia TR. Santos MLSC. ICNP® terminologica subgroup for palliative care patients with malignant tumor wounds. Acta Paul Enferm.2016;29(3):340-6. http://dx.doi.org/10.1590/1982-0194201600047.

9. Gonçalves MCDS, Brandão MAG, Duran ECM. Validation of the defining characteristics of the nursing diagnosis impaired comfort in oncology. Acta Paul Enferm. 2016;29(1):115-24. http://dx.doi.org/10.1590/19820194201600016.

10. LeDoux JE, Pine DS. Using neuroscience to help understand fear and anxiety: a two-system framework. Am J Psychiatry. 2016;173(11):1083-93. http://dx.doi.org/10.1176/appi.ajp.2016.16030353. PMid:27609244.

11. Herdman $\mathrm{H}$, Kamitsuru S. Diagnósticos de enfermagem da NANDA-I: definições e classificação - 2018/2020. 11. ed. Porto Alegre: Art Med;2018

12. Bousso RS, Poles K, Cruz DDALM. Nursing concepts and theories. Rev Esc Enferm USP. 2014;48(1):141-5. http://dx.doi.org/10.1590/S0080623420140000100018. PMid:24676120.

13. Lunney M.Uso do pensamento crítico para o alcance de resultados positivos de saúde. In: Lunney M. Pensamento crítico para o alcance de resultados 
positivos em saúde: análise e estudos de caso em enfermagem. Porto Alegre: Artmed; 2011. p. 25-55.

14. Craske MG, Stein MB, Eley TC, Milad MR, Holmes A, Rapee RM et al Correction: anxiety disorders. Nat Rev Dis Primers. 2017;3(1):17100. http:// dx.doi.org/10.1038/nrdp.2017.100. PMid:29239346.

15. Taylor-Loughran AE, O'Brien ME, LaChapelle R, Rangel S. Defining characteristics of the nursing diagnoses fear and anxiety: A validation study. Appl Nurs Res. 1989;2(4):178-86. http://dx.doi.org/10.1016/S08971897(89)80007-2.

16. Whitley GG, Tousman SA. A multivariate approach for validation of anxiety and fear. Int J Nurs Terminol Classif. 1996;7(3):116-24. http://dx.doi. org/10.1111/j.1744-618X.1996.tb00303.x. PMid:8868795

17. Suriano MLF, Michel JM, Zeitoun SS, Herdman TH, Barros ALBL. Consensual validation of the nursing diagnoses fear and anxiety identified at the immediate preoperative period in patients undergoing elective surgery. Int J Nurs Terminol Classif. 2011;22(3):133-41. http://dx.doi. org/10.1111/j.1744-618X.2011.01181.x. PMid:21777374.

18. Bay EJ, Algase DL. Fear and anxiety: a simultaneous concept analysis Int J Nurs Knowl. 1999;10(3):103-11. http://dx.doi.org/10.1111/j.1744618X.1999.tb00036.x. PMid:10595125.

19. Whitley GG. Concept analysis of fear. Int J Nurs Terminol Classif. 1992;3(4):155-61. http://dx.doi.org/10.1111/j.1744-618X.1992.tb00531.x. PMid:1489587.

20. Letho RH, Stein KF. Death anxiety: an analysis of an evolving concept: research and theory for nursing practice. Int J. 2009;23(1):23-41. http:// dx.doi.org/10.1891/1541-6577.23.1.23.

21. Costa JN, Lopes MVO, Moraes MHB. Simultaneous concept analysis of diagnoses related to urinary incontinence. Int J Nurs Knowl.2019;31(2):10923. http://dx.doi.org/10.1111/2047-3095.12254. PMid:31339006.

22. Haase JE, BrittT, Coward DD, Leidy NK, Penn PE. Simultaneous concept analysis of spiritual perspective, hope, acceptance and self-transcendence. Image J Nurs Sch. 1992;24(2):141-7.http://dx.doi.org/10.1111/j.1547-5069.1992. tb00239.x. PMid: 1601456.

23. Lopes MVO, Silva VM. Métodos avançados de validação de diagnósticos de enfermagem. In Herdman TH, organizador. PRONANDA Programa de atualização em diagnósticos de enfermagem: conceitos básicos. Porto Alegre: Artmed Panamericana; 2016. p. 87-132.

24. Mendes KDS, Silveira RCDCP, Galvão CM. Integrative literature review: a research method to incorporate evidence in health care and nursing Texto Contexto Enferm. 2008;17(4):758-64. http://dx.doi.org/10.1590/ S0104-07072008000400018.

25. Galica J, BrennenstuhI S, Maheu C, Townsley C, Metcalfe K. Examining the dimensionality of the Fear of Cancer Recurrence Inventory. Psychooncology. 2018;27(11):2602-8. http://dx.doi.org/10.1002/pon.4839. PMid:29998546.

26. Walker LO, Avant KC. Strategies for theory construction in nursing (6th ed.). Upper Saddle River: Pearson/Prentice Hall; 2018.

27. Martin P.The epidemiology of anxiety disorders: a review. Dialogues Clin Neurosci. 2003;5(3):281-98. PMid:22034470.

28. Oliveira N, Chianca T, Rassool GH. A validation study of the nursing diagnosis anxiety in Brazil. Int J Nurs Terminol Classif. 2008;19(3):102-10. http://dx.doi.org/10.1111/j.1744-618X.2008.00089.x. PMid:18798941.

29. Wein $S$, Sulkes A, Stemmer S. The oncologist's role in managing depression, anxiety, and demoralization with advanced cancer. Cancer J.2010;16(5):4939. http://dx.doi.org/10.1097/PPO.0b013e3181f28b64. PMid:20890146.

30. Stoklosa J, Patterson K, Rosielle D, Arnold RM. Anxiety in palliative care: causes and diagnosis\# 186. J Palliat Med. 2011;14(10):1173-4. http:// dx.doi.org/10.1089/jpm.2011.9644. PMid:22004149.

31. Castillo ARG, Recondo R, Asbahr FR, Manfro GG. Transtornos de ansiedade. Br J Psychiatry. 2000;22(suppl 2):20-3. http://dx.doi.org/10.1590/S151644462000000600006

32. Locker D. Psychosocial consequences of dental fear and anxiety. Community Dent Oral Epidemiol.2003;31(2):144-51. http://dx.doi.org/10.1034/j.16000528.2003.00028.x. PMid:12641596.

33. Finks K. Symptoms and treatment of anxiety disorders. NASN Sch Nurse. 2012;27(5):251-3. http://dx.doi.org/10.1177/1942602X12455950. PMid:23008867.

34. Gomes GLL, Nóbrega MML. Anxiety in children following hospitalization:a proposal for a nursing diagnosis. Rev Lat Am Enfermagem. 2015;23(5):96370. http://dx.doi.org/10.1590/0104-1169.0372.2637. PMid:26487148.
35. Gomes GLL, Fernandes MGM, Nóbrega MML. Hospitalization anxiety in children: conceptual analysis. Rev Bras Enferm. 2016;69(5):940-5. http:// dx.doi.org/10.1590/0034-7167-2015-0116. PMid:27783738.

36. Goyal AR, Bergh S, Engedal K, Kirkevold M, Kirkevold Ø. Anxiety, anxiety symptoms, and their correlates in persons with dementia in Norwegian nursing homes: a cause for concern. Dement Geriatr Cogn Disord. 2017;43(56):294-305. http://dx.doi.org/10.1159/000471796. PMid:28463828.

37. Humble M. Aetiology and mechanisms of anxiety disorders. Acta Psychiatr Scand. 1987;76(S335):15-30. http://dx.doi.org/10.1111/j.1600-0447.1987. tb07745.x. PMid:3314357.

38. Frederickson K. Using a nursing model to manage symptoms: anxiety and the Roy adaptation model. Holist Nurs Pract. 1993;7(2):36-43. http:// dx.doi.org/10.1097/00004650-199301000-00006. PMid:8429068.

39. Hilliard KM, Iwamasa GY. The conceptualization of anxiety: an exploratory study of Japanese American older adults. J Clin Geropsychol. 2001;7(1):5365. http://dx.doi.org/10.1023/A:1026416802568.

40. Koivula M, Paunonen-IImonen M, Tarkka MT, Tarkka M, Laippala P. Fear and anxiety in patients awaiting coronary artery bypass grafting. Heart Lung. 2001;30(4):302-11. http://dx.doi.org/10.1067/mhl.2001.116134. PMid:11449217.

41. Kilbride L, Smith G, Grant R. The frequency and cause of anxiety and depression amongst patients with malignant brain tumours between surgery and radiotherapy.J Neurooncol.2007;84(3):297-304. http://dx.doi. org/10.1007/s11060-007-9374-7. PMid:17594056.

42. Fardell JE, Thewes B, Turner J, Gilchrist J, Sharpe L, Smith A et al. Fear of cancer recurrence: a theoretical review and novel cognitive processing formulation. J Cancer Surviv.2016;10(4):663-73. http://dx.doi.org/10.1007/ s11764-015-0512-5. PMid:26782171.

43. Bayrampour H, Ali E, McNeil DA, Benzies K, MacQueen G, Tough S. Pregnancy-related anxiety: a concept analysis. Int J Nurs Stud. 2016;55:11530. http://dx.doi.org/10.1016/j.jpurstu.2015.10.023. PMid:26626973.

44. McNaughton N. What is anxiety and how should we treat it?'. NZ J Psycho [Internet]. 1996; [citado 2020 jun 10];25(1):51-61. Disponível em: https://www. researchgate.net/profile/Neil_Mcnaughton/publication/291904855_'What_is anxiety_and_how_should_we_treat_it'/links/56f992cc08ae38d710a3025c. pdf

45. Skaali T, Fosså SD, Bremnes R, Dahl O, Haaland CF, Hauge ER et al. Fear of recurrence in long-term testicular cancer survivors. Psychooncology. 2009;18(6):580-8. http://dx.doi.org/10.1002/pon.1437. PMid:18855944.

46. Vasconcelos ADS, Costa C, Barbosa LNF. Do transtorno de ansiedade ao câncer. Rev SBPH [Internet]. 2008; [citado 2020 jun 10];11(2):51-71. Disponível em: http://pepsic.bvsalud.org/pdf/rsbph/v11n2/v11n2a06.pdf

47. Bergamasco EC, Rossi LA, Carvalho EC, Dalri MCB. Diagnósticos de medo e ansiedade: validação de conteúdo para o paciente queimado. Rev Bras Enferm. 2004;57(2):170-7. http://dx.doi.org/10.1590/S003471672004000200008. PMid:15535515.

48. Rapee RM. Generalized anxiety disorder: a review of clinical features and theoretical concepts. Clin Psychol Rev. 1991;11(4):419-40. http://dx.doi. org/10.1016/0272-7358(91)90116-C.

49. Ziner KW, Sledge Jr GW, Bell CJ, Johns S, Miller KD, Champion VL Predicting fear of breast cancer recurrence and self-efficacy in survivors by age at diagnosis. Oncol Nurs Forum. 2012;39(3):287-95. http://dx.doi. org/10.1188/12.ONF.287-295. PMid:22543387.

50. Shay LA, Carpentier MY, Vernon SW. Prevalence and correlates of fear of recurrence among adolescent and young adult versus older adult posttreatment cancer survivors. Support Care Cancer. 2016;24(11):4689-96. http://dx.doi.org/10.1007/s00520-016-3317-9. PMid:27387913.

51. Moser DK. "The rust of life": impact of anxiety on cardiac patients. Am J Crit Care.2007;16(4):361-9. http://dx.doi.org/10.4037/ajcc2007.16.4.361. PMid:17595368.

52. Tramonti F, Maestri M, Gronchi A, Fabbrini M, Di Coscio E, Carnicelli Let al Psychological well-being of patients with insomnia and its relationship with anxiety and depression. Psychol Health Med. 2016;21(3):309-16. http:// dx.doi.org/10.1080/13548506.2015.1069856. PMid:26222934.

53. Taylor C, Richardson A, Cowley S. Surviving cancer treatment: an investigation of the experience of fear about, and monitoring for, recurrence in patients following treatment for colorectal cancer. Eur J Oncol Nurs. 2011;15(3):2439. http://dx.doi.org/10.1016/j.ejon.2011.03.010. PMid:21530395.

54. Armfield JM. Cognitive vulnerability: a model of the etiology of fear. Clin Psychol Rev.2006;26(6):746-68. http://dx.doi.org/10.1016/j.cpr.2006.03.007. PMid:16806621. 
55. Gomes ET, Souza Melo RLA, Vasconcelos EMR, Alencar EN. Uso dos diagnósticos de enfermagem ansiedade e medo nas clínicas médica e cirúrgica de um hospital universitário. Rev Pesquisa [Internet]. 2012; [citado 2020 jun 10];4(2):2419-26. Disponível em: https://www.redalyc. org/pdf/5057/505750893027.pdf

56. Calhoon GG, Tye KM. Resolving the neural circuits of anxiety. Nat Neurosci. 2015;18(10):1394-404. http://dx.doi.org/10.1038/nn.4101.PMid:26404714.

57. Whitley GG. Expert validation and differentiation of the nursing diagnoses anxiety and fear. Int J Nurs Terminol Classif. 1994;5(4):143-50. http://dx.doi. org/10.1111/j.1744-618X.1994.tb00263.x. PMid:7826717.

58. Fulton $\mathrm{CL}$. The physical and psychological symptoms experienced by patients with metastatic breast cancer before death. Eur J Cancer Care. 1997;6(4):262-6. http://dx.doi.org/10.1046/j.1365-2354.1997.00057.x. PMid:9460344.

59. Lebel S, Ozakinci G, Humphris G, Mutsaers B, Thewes B, Prins J et al. From normal response to clinical problem: definition and clinical features of fear of cancer recurrence. Support Care Cancer. 2016;24(8):3265-8. http://dx.doi.org/10.1007/s00520-016-3272-5. PMid:27169703.

60. Sharpley CF, Christie DR. 'How I was then and how I am now': current and retrospective self-reports of anxiety and depression in Australian women with breast cancer. Psychooncology. 2007;16(8):752-62. http://dx.doi. org/10.1002/pon.1125. PMid:17089443.

61. Holanda VN, Bezerra AS, Tavares AR, Lima CIR, Mamede LTS, Araújo RLQ et al. As bases biológicas do medo: uma revisão sistemática da literatura. Rev Interfaces [Internet]. 2013; [citado 2020 jun 10];1(3):1-15 Disponível em: http://interfaces.leaosampaio.edu.br/index.php/revistainterfaces/article/viewFile/419/300

62. Esperidião-Antonio V, Majeski-Colombo M, Toledo-Monteverde D, MoraesMartins G, Fernandes JJ, Assis MBD et al. Neurobiologia das emoções. Archives of Clinical Psychiatry.2008;35(2):55-65. http://dx.doi.org/10.1590/ S0101-60832008000200003.

63. Brandão ML. As bases biológicas do comportamento: introdução à neurociência. Rev Inst Med Trop São Paulo. 2005;47(3):124. http://dx.doi. org/10.1590/S0036-46652005000300013.

64. Menezes GB, Fontenelle LF, Mululo S, Versiani M. Treatmentresistant anxiety disorders: social phobia, generalized anxiety disorder and panic disorder. Rev Bras Psiquiatr. 2007;29(2, Suppl 2):S55-60. http://dx.doi.org/10.1590/S1516-44462007000600004. PMid:18157434. 